\title{
Ecology in a changing climate
}

\section{Complex ecological and evolutionary controls of forest dynamics make projecting the future difficult.}

For hundreds of thousands of years, trees have provided all kinds of benefits to humanity. Millions of us use wood as fuel, we rely on their fruits and oils, and our houses are woodframed. We climb them as children, and sit in their shade to escape the heat. But in the past few decades, our focus has increasingly shifted to the importance of trees in regulating regional hydrology ${ }^{1}$, global climate ${ }^{2}$ and the global carbon cycle ${ }^{3}$ as we continue to pump greenhouse gases into the atmosphere. Studies published in this issue (page 441) and the past six months in Nature Geoscience ${ }^{4-8}$ highlight that some of the environmental factors thought to control forest dynamics and productivity act in ways quite different than we might have expected, or sometimes not at all.

Terrestrial ecosystems store about three times as much carbon as resides in the atmosphere, and forests are the largest terrestrial carbon $\sin ^{3}$. Understanding what regulates the carbon dynamics of forests brings us into the complex, knotty realm of ecology. A tangle of different factors control the growth of a plant and the dynamics of a plant community: the availability of nutrients such as nitrogen or iron, temperature, ozone and $\mathrm{CO}_{2}$ concentrations, water availability, competition and herbivory, and the biodiversity present in a community.
There is still much to understand about the individual elements in this tangle. We are finding surprises even in relationships we thought we understood fairly well. For example, evidence that tropical tree growth may not be fertilized by increasing atmospheric $\mathrm{CO}_{2}$ concentrations ${ }^{4}$ went against expectations. But we have even more to learn about the potentially complex interactions among a variety of environmental and ecological factors. These interactions can lead to unexpected effects. For example, $\mathrm{CO}_{2}$, water, and nitrogen act in a variety of combinations to jointly limit plant productivity in a grassland ${ }^{5}$.

The ecological controls over forest carbon dynamics can be intertwined with the evolutionary strategies that species employ. For example, species that have evolved to survive and deter the spread of forest fires predominate in Eurasian boreal forests. But in North America, the dominant species burn readily, fuel more intense forest fires and create landscapes free of competition where new seedlings thrive ${ }^{6}$. Evolution shapes these differences, which result in distinct regional climate feedbacks.

Rapid ongoing changes to the regional and global environment are already having noticeable effects on the ecology of forests.
Models remain some of our best tools for projecting how these changes may feed back, although it is difficult to constrain ecological complexities sufficiently well to confidently incorporate them into regional and global models. So researchers have had to simplify. For example, most global vegetation models exclude factors such as nitrogen or phosphorus limitation in their simulations of the global carbon cycle. As a consequence, these simulations can vastly overestimate the amount of carbon taken up and stored by forests in the future (page 441).

In the models, it will be impossible to factor in all the intricate interactions between biological, chemical and physical components of ecosystems. We must keep working to identify the most significant ones, and tractable ones such as physiologically based drought thresholds of tree mortality ${ }^{8}$. Then we just have to ensure that our more dire projections don't come to pass.

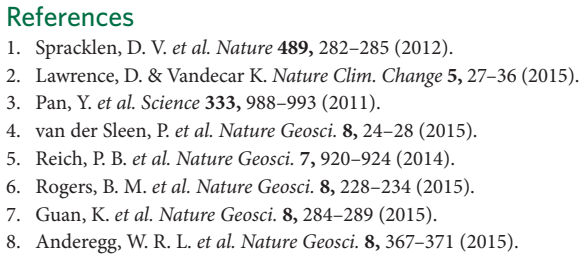

\section{More space for methods}

\section{Nature Geoscience introduces 3,000-word Methods sections that are integrated with the online paper.}

Up to now, methodological details within Nature Geoscience papers had to be kept brief. More detail could be provided in Supplementary Information files online, but with the drawback that they need to be opened separately, and that references in this material are not counted as citations by indexing services such as Scopus or Thomson Web of Science. We are now addressing these shortcomings by offering more space for Methods and their references within the online paper, as an integral part of both the HTML and PDF versions (see http://dx.doi. org/10.1038/ngeo2438 for an example).

Scientific papers, and the ways they are being read, have changed dramatically. Physical copies of the journal have become much less important as almost all scientists access, read and file their scientific intake online. At the same time, more rigour and detail in methods descriptions is required, as well as acknowledgement and traceability of source material. So it makes sense to offer fully integrated online-only space for better documentation of methods within the article.

Unlike Supplementary Information, online-only methods will be copy-edited. Importantly, online-only methods can contain all the relevant references fully accounted for by the indexing services. There is a limit of 3,000 words, not counting the reference list: we cannot offer copy-editing on unlimited amounts of text. But keep in mind that it is in the interest of both readers and referees to keep the Methods concise, without compromising on completeness. We feel that for most of the brief articles we publish, this space should be sufficient.
Supplementary Tables and Figures are not accommodated within the article in an online-only fashion; these will continue to be published in separate files as Supplementary Information.

We do not want to lose flexibility. Therefore for rare exceptions - for example, where supplementary methods and supplementary figures cannot easily be separated - we continue to offer the option of methods descriptions and other text material in Supplementary Information. But we appeal to our authors to think twice before they opt to separate the information on how they obtained their conclusions from the paper itself. In the vast majority of cases, we feel that readers - and authors of any cited papers are better served by an extended online-only Methods section. 